\title{
VECTOR FIELDS IN THE VICINITY OF A CIRCLE OF CRITICAL POINTS
}

\section{J. F. MATTEI AND M. A. TEIXEIRA}

\begin{abstract}
In this paper the $C^{S}$-conjugacy between vector fields on $\mathbf{R}^{2}$ having a circle of critical points is studied.
\end{abstract}

0. Statement of the problem. We are interested in studying vector fields on $\mathbf{R}^{2}$ having a circle of critical points as well as diffeomorphisms on $\mathbf{R}^{2}$ having a circle of fixed points. We can produce examples of such vector fields (resp. diffeomorphisms) by blowing up in polar coordinates germs of $C^{\infty}$ vector fields (resp. diffeomorphisms) on $\mathbf{R}^{2}$ at $\mathbf{0}$ having the form

$$
\tilde{X}(x, y)=\left(x^{2}+y^{2}\right)^{k}\left[(a x-b y) \frac{\partial}{\partial x}+(b x+a y) \frac{\partial}{\partial y}\right],
$$

with $b \neq 0$ and $k$ being a positive integer (resp. $\tilde{\varphi}=\operatorname{Id}+\tilde{X}$ ).

A natural question in the study of singularities is: "Under which conditions is a germ of a singularity $C^{s}$-determined by a finite jet and which finite jets are determining?" On $\mathbf{R}^{2}$ there is the following result due to F. Dumortier [1]:

If a germ satisfies an inequality of Lojawiewicz and has a characteristic orbit, then it is $C^{0}$-determined. (By "characteristic orbit" we mean an orbit which tends to the singularity or leaves the singularity with a well-defined direction. Observe that the field given above does not have a characteristic orbit.)

Consider germs of vector fields in $\mathbf{R}^{2}$ at $\mathbf{0}$ having the form

$$
\bar{Z}(x, y)=r^{k} \cdot \bar{X}(x, y)
$$

where $r^{2}=x^{2}+y^{2}, k$ is a positive integer and $\bar{X}$ is a germ of $C^{\infty}$ vector fields at 0 with $\bar{X}(0)=0$ and the eigenvalues of $\bar{X}^{\prime}(0)$ are $\lambda=a \pm i b$ with $a \neq 0$ and $b \neq 0$.

Consider the germs of diffeomorphisms in the plane of the form $\bar{\varphi}=\operatorname{Id}+\bar{Z}$. Denote by $Z$ and $\varphi$ the respective blowing ups (in polar coordinates) of $\bar{Z}$ and $\bar{\varphi}$.

Call $X^{k}$ and $D^{k}$ the spaces of such $Z$ and $\varphi$, respectively, both endowed with the $C^{s}$-topology $(s>k)$.

We recall that:

(i) The number $\alpha=a b^{-1}$ is a topological invariant for the structural stability in $D^{2}$ (see for instance [6]).

(ii) The diffeomorphism $\bar{\varphi}$ is formally imbedded in a flow represented by a vector field $\hat{H}$. Moreover the $(k+1)$-jet of $\hat{H}$ at 0 is $\hat{H}_{k}=\left(x^{2}+y^{2}\right) \bar{X}_{0}(x, y)$, where $\bar{X}_{0}$ is the linearized vector field of $\bar{X}$ (see [4]).

Received by the editors June 20, 1985.

1980 Mathematics Subject Classification. Primary 58F14; Secondary 34C35.

Key words and phrases. Vector field, critical point, conjugacy. 
(iii) Let $\varphi$ and $\psi$ be in $D^{2}$ such that both $\bar{\varphi}$ and $\bar{\psi}$ are imbedded in flows $\bar{H}$ and $\bar{G}$, respectively. A conjugacy between $H$ and $G$ implies that $\varphi$ and $\psi$ are also conjugate.

Consider now $\bar{Z}=r^{k} \bar{X}(x, y)$ and $\bar{Z}_{0}=r^{k} \bar{X}_{0}(x, y)$, with $\bar{X}$ and $\bar{X}_{0}$ as above. As before, let $Z$ and $Z_{0}$ be the elements of $X^{k}$ obtained from $\bar{Z}$ and $\bar{Z}_{0}$, respectively.

In this paper we treat the question of $C^{l}$-conjugacy between $Z$ and $Z_{0}$. Our main results concern the formal and $C^{l}$-determinacy of vector fields of the form:

$$
X(r, \theta)=\left(\mathbf{C} r^{k+1}+r^{k+2} a(r, \theta)\right) \frac{\partial}{\partial r}+\left(S r^{k}+r^{k+1} b(r, \theta)\right) \frac{\partial}{\partial \theta}
$$

in the vicinity of $S^{\prime}=0 \times S^{1}$, where $\mathbf{C}, S$ are scalars with $\mathbf{C} S \neq 0$ and $a(r, \theta)$, $b(r, \theta)$ are $C^{\infty}$ periodic functions in the variable $\theta$.

It will be shown that a conjugacy always exists and this conjugacy is $C^{\infty}$ provided the original vector field is nonresonant. The resonance condition is identified by the construction of a formal conjugacy between the vector fields.

In the case that the original vector field is resonant, a $C^{l}$-conjugacy can be constructed.

It should be mentioned that Dumortier and Roussarie [2] have dealt with the finite determining of germs of vector fields on $\mathbf{R}^{2}$ having the following normal form:

$$
X(r, \theta)=\left(\phi_{1}(\theta) r^{k+1}+O\left(r^{k+2}\right)\right) \frac{\partial}{\partial r}+\left(\alpha(\theta)+r^{k} \phi_{2}(\theta)+O\left(r^{k+1}\right)\right) \frac{\partial}{\partial \theta}
$$

for certain functions $\theta_{1}, \theta_{2}$ and $\alpha$. We emphasize that the techniques and methods used there are quite different from ours.

1. Preliminaries and notations. Let $X$ be a germ of a $C^{\infty}$ vector field at 0 in $\mathbf{R}^{2}$, such that its $\infty$-jet is given by

$$
\hat{X}(x, y)=r^{k} \Gamma \cdot\left(\begin{array}{l}
x \\
y
\end{array}\right)+\left(\begin{array}{l}
A(x, y) \\
B(x, y)
\end{array}\right)
$$

where $k=2,3,4,5, \ldots, n, \ldots, r^{2}=x^{2}+y^{2}, A(x, y)=\sum_{i+j \geq k+2} A_{i j} x^{i} y^{j}, B(x, y)=$ $\sum_{i+j \geq k+2} B_{i j} x^{i} y^{j}$, and $\Gamma=\rho R_{\alpha}$, where $R_{\alpha}$ is the rotation

$$
R_{\alpha}=\left(\begin{array}{cc}
\cos \alpha & \sin \alpha \\
-\sin \alpha & \cos \alpha
\end{array}\right) \text {. }
$$

Assume that $\alpha \notin \pi / 2 \cdot \mathbf{Z}$. Let

$$
E: \mathbf{R} \times S^{1} \rightarrow \mathbf{R}^{2}, \quad(r, \theta) \rightarrow(r \cos \theta, r \sin \theta) .
$$

Then we can define a $C^{\infty}$ vector field $\hat{X}$ on $\mathbf{R} \times S^{1}$ with $E_{*}(X)=\hat{X} \circ E$.

Let $\chi^{\infty}\left(\mathbf{R} \times S^{1} ; 0\right)$ be the space of germs of $C^{\infty}$ vector fields along $S^{1}=S^{1} \times 0$. This space is identified with $\chi_{2 \pi}^{\infty}\left(\mathbf{R}^{2}\right)$, the space of germs of $C^{\infty}$ vector fields along the $\theta$-axis, $\mathbf{R}_{\theta}=0 \times S^{1}$, which are periodic in the variable $\theta$, with period $2 \pi$.

Also we identify the ring $\xi_{0}$ of germs of $\mathbf{C}^{\infty}$ functions along $S^{1}$ with the ring $C_{2 \pi}^{\infty}$ of germs of $C^{\infty}$ functions along $\mathbf{R}_{\theta}$, periodic of period $2 \pi$ in the variable $\theta$.

Let $\hat{\mathcal{P}}=\mathbf{R}[\cos \theta, \sin \theta][[r]]$ be the ring of formal power series in $r$ whose coefficients are trigonometric polynomials.

Of course, we may consider $A^{\prime}=A \circ E$ and $B^{\prime}=B \circ E$ as elements of $\hat{P}$. 
Consider $\hat{\xi}_{0}=C^{\infty}\left(S^{1}\right)[[r]]$ as the ring of formal power series in $r$ with coefficients in $C_{2 \pi}^{\infty}$.

The elements of the space

$$
\hat{\chi}_{2} \simeq \hat{\xi}_{0} \partial / \partial r \oplus \hat{\xi}_{0} \partial / \partial \theta
$$

are called transversally formal germs of vector fields along $S^{1}$.

Let $G$ (resp. $\hat{G}$ ) be the group of diffeomorphisms along $S^{1}$ of the form $\phi=$ $\left(r+r^{2} q_{1}, \theta+r g_{2}\right)$ with $g_{1}, g_{2} \in \xi_{0}$ (resp. $\left.g_{1}, g_{2} \in \hat{\xi}_{0}\right)$.

Finally we define

$$
\hat{X}^{\prime}=\hat{P} \partial / \partial r \oplus \hat{P} \partial / \partial \theta
$$

and

$$
\hat{G}^{\prime}=\left\{\left(r+r^{2} g_{1}, \theta+r g_{2}\right) \text { such that } g_{1}, g_{2} \in \hat{\mathcal{P}}\right\} .
$$

2. Statement of the results.

(2.1) Theorem 1 (Formal CONJUGaCY). Consider $X \in \hat{X}_{2 \pi}$ (resp. $X_{2 \pi}^{\prime}$ ) given by

$$
X(r, \theta)=\left(\mathbf{C} r^{k+1}+r^{k+2} a\right) \frac{\partial}{\partial r}+\left(S r^{k}+r^{k+1} b\right) \frac{\partial}{\partial \theta}
$$

such that $a, b \in \hat{\xi}_{0}$ (resp. $a, b \in \hat{\mathcal{P}}$ ) and $\mathbf{C}, S$ are scalars with $C S \neq 0$. Then there exists a diffeomorphism $\varphi \in \hat{G}$ (resp. $\left.\varphi \in G^{\prime}\right)$ such that

$$
\varphi_{*}(X)=\left(\mathbf{C} r^{k+1}+c_{2 k+1} r^{2 k+1}\right) \frac{\partial}{\partial r}+S r^{k} \frac{\partial}{\partial \theta},
$$

where $c_{2 k+1}$ is a constant which depends only on the $(k+1)$-jets of $a(r, \varphi)$ and $b(r, \varphi)$.

(2.2) TheOREM 2 ( $C^{l}$-CONJUGaCY). Consider $X \in X_{2 \pi}^{\infty}$ given by

$$
X(r, \theta)=\left(\mathbf{C} r^{k+1}+r^{k+2} a\right) \frac{\partial}{\partial r}+\left(S r^{k}+r^{k+1} b\right) \frac{\partial}{\partial \theta}
$$

such that $\mathbf{C}, S$ are nonzero scalars, $a, b \in \xi_{0}$, and $k>2$. Then there exists $a$ $\mathbf{C}^{l}$-diffeomorphism on a neighborhood of $S^{1}$ in $\mathbf{R} \times S^{1}$ of the form

$$
\phi(R, \varphi)=\left(R+R^{2} u(R, \varphi), \varphi+R v(R, \varphi)\right)
$$

such that

(i) $l=k-2$,

(ii) $\phi_{*}(X)=\mathbf{C} R^{k+1} \partial / \partial R+S R^{k} \partial / \partial \varphi$,

(iii) if $X$ is formally conjugate to $X_{0}=\mathbf{C} r^{k+1} \partial / \partial r+S r^{k} \partial / \partial \theta$, then $\phi$ can be chosen to be a $C^{\infty}$-diffeomorphism.

One deduces the following corollary:

(2.3) COROLLARY. Let $X$ be a germ of a $C^{\infty}$ vector-field at 0 in $\mathbf{R}^{2}$ given by

$$
Z(x, y)=r^{k} X(x, y),
$$


where $r^{2}=x^{2}+y^{2}, k>2$, and the eigenvalue of the linear part of $X(x, y)($ at 0$)$ are $\lambda=a \pm i b$ with $a b \neq 0$. Then $Z$ is $C^{l}$-conjugate to $Z_{0}=r^{k} X_{0}$, where $X_{0}$ is the linearization of $X$ at 0 and $l \leq(k-1) / 2$.

3. Proof of Theorem 1. Let $\phi=(r(R, \varphi), \theta(R, \varphi))$ be the formal diffeomorphism given by

$$
r(R, \varphi)=R+u(R, \varphi), \quad \theta(R, \varphi)=\varphi+v(R, \varphi),
$$

where

$$
u=\sum_{j \geq 2} u_{j}(\varphi) R^{j}, \quad v=\sum_{j \geq 1} v_{j}(\varphi) R^{j}
$$

with $u_{j}, v_{j} \in C^{\infty}\left(S^{1}\right)$ (resp. they are trigonometric polynomials).

The field $\phi_{*}(X)=(D \phi)^{-1} \circ X \circ \phi$ has the expression

$$
\dot{R}=\frac{d R}{d t}=C R^{k+1}+R^{k+2} A, \quad \dot{\varphi}=\frac{d \varphi}{d t}=S R^{k}+R^{k+1} B,
$$

where

$$
A=A(R, \varphi)=\sum_{j \geq 0} A_{j}(\varphi) R^{j}, \quad B=B(R, \varphi)=\sum_{j \geq 0} B_{j}(\varphi) R^{j} .
$$

Combining (3.1), (3.2) and (2.1.1) one has

$$
\left\{\begin{array}{c}
\left(1+u_{R}\right)\left(\mathbf{C} R^{k+1}+R^{k+2} A\right)+u_{\varphi}\left(S R^{k}+R^{k+1} B\right) \\
=\mathbf{C}(R+u) R^{k+1}+R^{k+2} a(R+u, \varphi+v) \\
v_{R}\left(\mathbf{C} R^{k+1}+R^{k+2} A\right)+\left(1+v_{\varphi}\right)\left(S R^{k}+R^{k+1} B\right) \\
=S(R+u)^{k}+R^{k+1} b(R+u, \varphi+v) .
\end{array}\right.
$$

Identifying the respective coefficients of $R^{j+k}$ in (3.3), we get (3.4)

$$
\left\{\begin{array}{l}
(j-k-1) \mathbf{C} u_{j}+S\left(u_{j}\right)_{\varphi}=a_{j-2}-A_{j-2}+P_{j}\left(u_{2}, \ldots, u_{j-1}, v_{1}, \ldots, v_{j-2}\right), \\
j \mathbf{C} v_{j}+S\left(v_{j}\right)_{\varphi}=b_{j-1}-B_{j-1}+Q_{j}\left(u_{2}, \ldots, u_{j+1}, v_{1}, \ldots, v_{j-1}\right),
\end{array}\right.
$$

where $P_{j}$ and $Q_{j}$ are polynomials in $u_{k}, v_{i}$ and also depending on $A_{0}, \ldots, A_{j-3}$ and $B_{0}, \ldots, B_{j-2}$.

We may rewrite (3.4) as follows:

$$
(3.5)_{j+1}\left\{\begin{array}{c}
(j-k) \mathbf{C} u_{j+1}+S\left(u_{j+1}\right)_{\varphi} \\
=a_{j-1}-A_{j-1}+P_{j+1}\left(u_{2}, \ldots, u_{j}, v_{1}, \ldots, v_{j-1}\right), \\
j \mathbf{C} v_{j}+S\left(v_{j}\right)_{\varphi}=b_{j-1}-B_{j-1}+Q_{j}\left(u_{2}, \ldots, u_{j+1}, v_{1}, \ldots, v_{j-1}\right) .
\end{array}\right.
$$

Because of Lemma (3.9) (see below), one may chose $u_{j}$ and $v_{j}$ in such a way that $A_{j}=0$ if $j \neq k-1, B_{j}=0$ for any $j$ and $A_{k-1}$ is a suitable constant. We claim that it must satisfy the equation:

$$
\int_{0}^{2 \pi}\left(a_{k-1}-A_{k-1}+P_{k+1}\right) d \varphi=0
$$

which is determined by formula $(3.5)_{k+1}$. 
(3.7) REMARK. The above equality (3.6) characterizes the resonance which occurs in the assertion of Theorem 1.

Consider, for each periodic function $h(\varphi)$ (resp. $h \in \mathbf{R}[\sin \varphi, \cos \varphi]$ ), the differential equation

$$
\overline{\mathbf{C}} \cdot W+S W_{\varphi}=h(\varphi)
$$

We shall omit the proof of the next lemma.

(3.9) LEMMA. Assume that either (i) $\overline{\mathbf{C}} \neq 0$ or (ii) $\overline{\mathbf{C}}=0$ and $\int_{0}^{2 \pi} h(\varphi) d \varphi=$ 0. Then equation (3.6) admits a periodic solution $w(\varphi)($ resp. $w \in \mathbf{R}[\sin \varphi, \cos \varphi])$.

From (3.2) and (3.6) we get the following normal form for $X$

$$
\dot{R}=\mathrm{C} R^{k+1}+c_{2 k+1} R^{2 k+1}, \quad \dot{\varphi}=S R^{k},
$$

where $c_{2 k+1} \in \mathbf{R}$ is given by the expression

$$
c_{2 k+1}=\frac{1}{2 \pi} \int_{0}^{2 \pi}\left(a_{k-1}+P_{k+1}\right) d \varphi
$$

This finishes the proof of Theorem 1.

\section{Proof of Theorem 2.}

(4.0) Some comments. Before proving Theorem 2 we shall give an intuitive idea of the strategy of the proof. First of all, we seek a diffeomorphism $\varphi=$ Id $+H$ such that: (i) $\varphi_{*}(X)$ is the required normal form and (ii) $H(R, \varphi)=$ $\left(R^{2} U(R, \varphi), R V(R, \varphi)\right)$. The problem is then reduced to answering the following question: "Given $C^{l}$-periodic (in $\varphi$ ) mappings $a_{1}(R, \varphi), a_{2}(R, \varphi)$, do there exist $C^{l}$-periodic mappings $U(R, \varphi), V(R, \varphi)$ which are solutions of

$$
\left\{\begin{array}{l}
\mathbf{C} R U_{R}+S U_{\varphi}=(k-1) U+a_{1}\left(R-R^{2} U, \varphi+R V\right), \\
R V_{R}+S V_{\varphi}=k S U-\mathbf{C} V+a_{2}\left(R+R^{2} U, \varphi+R V\right) ? "
\end{array}\right.
$$

Set $g_{i}(R, \varphi, U, V)=a_{i}\left(R+R^{2} U, \varphi+R V\right), i=1,2$.

The answer to this question depends on the Fundamental Lemma and Proposition (4.12) (see below). The proof of the Fundamental Lemma is based essentially on the Characteristical Line Method applied to equations (4.0.1). It says that given $C^{l}$-periodic functions $h_{1}, h_{2}$ one can find $C^{l}$-periodic functions $U=Q_{1}\left(h_{1}, h_{2}\right)$, $V=Q_{2}\left(h_{1}, h_{2}\right)$ which are solutions of

$$
\left\{\begin{array}{l}
R U_{R}+S U_{\varphi}=\mathbf{C}(k-1) V+h_{1}, \\
R V_{R}+S V_{\varphi}=k S U-\mathbf{C} V+h_{2}, \quad \text { for } R \text { small. }
\end{array}\right.
$$

Moreover the expression for $Q_{1}\left(h_{1}, h_{2}\right)$ and $Q_{2}\left(h_{1}, h_{2}\right)$ are given explicitly.

So it remains to look for those $U, V$ such that

$$
\left\{\begin{array}{l}
U(R, \varphi)=Q_{1}\left(g_{1}(R, \varphi, U(R, \varphi), V(R, \varphi))\right), \\
V(R, \varphi)=Q_{2}\left(g_{2}(R, \varphi, U(R, \varphi), V(R, \varphi))\right), \quad \text { for } R \text { small. }
\end{array}\right.
$$

We define for each $(R, \varphi)$ the functionals

$$
P_{1}(U, V)=Q_{1}\left(g_{1}(R, \varphi, U, V)\right), \quad P_{2}(U, V)=Q_{2}\left(g_{2}(R, \varphi, U, V)\right) .
$$

Finally one proves that $P=\left(P_{1}, P_{2}\right)$ is invertible. 
Proposition (4.12) is concerned with the $C^{l}$-extension to $\mathbf{R}^{2}$ of mappings obtained by the Fundamental Lemma.

We fix some notation:

$C_{2 \pi}^{l}(\Omega)$ is the space of $\mathbf{C}^{l}$ functions $f: \Omega \times \mathbf{R} \rightarrow \mathbf{R}$ periodic of period $2 \pi$ in the second variable, with $\Omega \subset \mathbf{R}^{n}$.

Assuming the above notation we set

$C_{2 \pi}^{l}=C_{2 \pi}^{l}(\mathbf{R})$.

$\left(C_{2 \pi}^{l}\right)_{0}$ is the subspace of $C_{2 \pi}^{l}$ constituted by the functions $f$, such that $\operatorname{supp}(f) \subset$ $[-\rho,+\rho] \times \mathbf{R}$ for some $\rho>0$, i.e. $f$ defines a function with compact support on $\mathbf{R} \times S^{1}$.

If $r \leq s$ and $\rho>0$, then

$\|f\|_{r, \Omega}=\sup \left\{\left|D_{x}^{\alpha} f\right| ; x \in \Omega\right.$ and $\left.|\alpha| \leq r, \alpha=\left(\alpha_{1}, \ldots, \alpha_{k}\right)\right\}$,

$\|f\|_{r}=\|f\|_{r, \mathbf{R}} m$,

$\|f\|_{r, \rho}=\|f\|_{r, \beta(\rho)}$, where $B(\rho)=\left\{\left(x_{1}, \ldots, x_{n}\right) ;\left|x_{1}\right| \leq \rho\right\}$.

(4.1) REMARK. Let $f_{1}, f_{2}$ be $C^{\infty}$ functions defined in some open domains of $\mathbf{R}^{n}$. It is easy to see that

$$
\left\|f_{1} \circ f_{2}\right\|_{r, E} \leq N_{r}\left\|f_{1}\right\|_{r, f_{2(E)}}\left(1+\left\|f_{2}\right\|_{r, E}\right)^{r},
$$

where $N_{r}$ depends only on $r$.

(4.2) Fundamental Lemma. Let $\mathbf{C}, S, \overline{\mathbf{C}}$ be nonzero scalars and $l \in \mathbf{N} \cup$ $\{\infty\}$. Given $h(R, \varphi)$ in $C_{2 \pi}^{l}$ consider the equation

$$
\mathbf{C} R U_{R}+S U_{\varphi}=\overline{\mathbf{C}} U+h .
$$

Then this equation admits a solution $U(R, \varphi)$ in $C_{2 \pi}^{l}$ provided that one of the following conditions is satisfied:

(a) $\overline{\mathbf{C}} / \mathbf{C}<0$.

(b) $\overline{\mathbf{C}} / \mathbf{C} \geq l+1$ and $h$ has support on $\Omega_{0}=\left[-\rho_{0}, \rho_{0}\right] \times \mathbf{R}$, with $\rho_{0}>0$.

Moreover

(a) If $\overline{\mathbf{C}} / \mathbf{C}<0$, then there exists a linear transformation

$$
L_{\mathbf{C}, S, \overline{\mathbf{C}}}: C_{2 \pi}^{l} \rightarrow C_{2 \pi}^{l}
$$

such that for any $r \leq l, r<\infty$, one can define a constant $K_{\mathbf{C}, S, \overline{\mathbf{C}}}^{(r)}>0$ which satisfies:

(a $\left.\mathbf{a}_{\mathbf{1}}\right) L_{\mathbf{C}, S, \overline{\mathbf{C}}}(h)$ is a solution of (4.2.1).

$\left(\mathrm{a}_{2}\right)\left\|L_{\mathbf{C}, S, \overline{\mathbf{C}}}(h)\right\|_{r, T_{\rho}} \leq K_{\mathbf{C}, S, \overline{\mathbf{C}}}^{(r)}(1+\rho)^{2 r}\|h\|_{r, T_{\rho}}$, where $T_{\rho}=\left\{(R, \varphi) \in \mathbf{R}^{2} ;|R| \leq e^{-\mathbf{C} \varphi},|\varphi| \leq 2 \pi\right\}$.

(b) If $l<\infty$ and $\mathbf{C} / \mathbf{C} \geq l+1$, then there exist a linear transformation

$$
L_{\mathbf{C}, S, \overline{\mathbf{C}}}:\left(C_{2 \pi}^{l}\right)_{0} \rightarrow C_{2 \pi}^{l}
$$

and a constant $K_{\mathbf{C}, S, \overline{\mathbf{C}}}>0$ such that:

$\left(\mathrm{b}_{1}\right) L_{\mathbf{C}, S, \overline{\mathbf{C}}}(h)$ is a solution of (4.2.1).

$\left(\mathrm{b}_{2}\right)\left\|L_{\mathbf{C}, S, \overline{\mathbf{C}}}\right\|_{l, T_{\rho}} \leq K_{\mathbf{C}, S, \overline{\mathbf{C}}}(1+\rho)^{2 l}\|h\|_{l}$.

Proof of Lemma (4.2). It is sufficient to prove this lemma in the case $\overline{\mathbf{C}}<0$ and $S=1$. The remaining cases follow easily. 
Observe that the vector field $\mathbf{Z}=\mathbf{C} R \partial / \partial R+\partial / \partial \varphi$ is transformed by the diffeomorphism (on $\left.\mathbf{R}^{2}\right) \xi:(R, \varphi) \rightarrow\left(R e^{\mathbf{C} \varphi}, \varphi\right)$ into $\xi_{*}(Z)=\partial / \partial \varphi$.

In the same way equation (4.2.1) is transformed into

$$
W_{\varphi}=\overline{\mathbf{C}} W+H(R, \varphi), \quad \text { where } W=U \circ \xi \text { and } H=h \circ \xi \text {. }
$$

Moreover the diffeomorphism transforms $C_{2 \pi}^{l}$ in the space $C_{Z}^{l}$ of the $\mathbf{C}^{l}$-functions $f(R, \varphi)$ on $\mathbf{R} \times S^{1}$ which verify

$$
f\left(R e^{-2 \pi \mathbf{C}}, \varphi+2 \pi\right)=f(R, \varphi) .
$$

The solutions of (4.2.2) are the functions

$$
W(R, \varphi)=W_{0}(R) e^{\overline{\mathbf{C}} \varphi}+\left(\int_{0}^{\varphi} e^{-\overline{\mathbf{C}} s} H(R, s) d s\right) e^{\overline{\mathbf{C}} \varphi} .
$$

Observe that $W$ must satisfy (4.3). This means that the function

$$
\Delta(R, \varphi)=W\left(R e^{-2 \pi \mathrm{C}}, \varphi+2 \pi\right)-W(R, \varphi)
$$

must be zero.

Since $H$ verifies (4.2.2), one has $\partial \Delta / \partial \varphi=\overline{\mathbf{C}} \Delta$ and so $\Delta(R, \varphi)=\Delta(R, 0) e^{\overline{\mathbf{C}} \varphi}$.

From (4.4) and (4.5) we get the relation

$$
e^{2 \pi \overline{\mathrm{C}}} W_{0}\left(R e^{-2 \pi \mathrm{C}}\right)-W_{0}(R)=-F(R),
$$

where $F(R)=\left(\int_{0}^{2 \pi} e^{-\overline{\mathrm{C}} s} H(R, s) d s\right) e^{2 \pi \overline{\mathrm{C}}}$. We write

$$
W_{0}(R)=\sum_{j=0}^{\infty} e^{2 \pi j \overline{\mathbf{C}}} F\left(e^{-2 \pi j \mathrm{C}} R\right) .
$$

We are going to separate the cases:

Case A. Assume $\mathbf{C}>0$; this is just the first assumption of the preceding lemma. The series in consideration, (4.7), converges, as well as its derivatives

$$
W_{0}^{(\alpha)}(R)=\sum_{j=0}^{\infty} e^{2 \pi j(\overline{\mathbf{C}}-\alpha \mathbf{C})} F^{(\alpha)}\left(e^{-2 \pi j \mathbf{C}} R\right) .
$$

Observe that, for $r \leq l$ and $r<\infty$, one has

$$
\left\|W_{0}\right\|_{r, \rho} \leq \frac{1}{1-e^{2 \pi \overline{\mathrm{C}}}}\|F\|_{r, \rho} .
$$

On the other hand, it is easy to check that

$$
\|W\|_{r, \Sigma_{\rho}} \leq K_{r}^{\prime}\|H\|_{r, \Sigma_{\rho}},
$$

where $\Sigma_{\rho}=[-\rho,+\rho] \times[-2 \pi, 2 \pi]$ and $K_{r}^{\prime}$ depends only on $r$.

We try to obtain similar inequalities for $U$ and $H$.

Observe that $T_{\rho}=\xi\left(\Sigma_{\rho}\right)$. And so, by inequality (4.1.1) we have

$$
\begin{gathered}
\|\xi\|_{r, \Sigma_{\rho}} \leq(1+|\mathbf{C}|)^{r} e^{2 \pi|\mathbf{C}|} \rho \\
\left\|\xi^{-1}\right\|_{T_{\rho}} \leq(1+|\mathbf{C}|)^{r} e^{2 \pi|\mathbf{C}|} \rho .
\end{gathered}
$$


Combining (4.9), (4.10) and (4.11) we get a number $K_{\mathbf{C}, S, \overline{\mathbf{C}}}^{(r)}$ depending only on $r, \mathbf{C}, S, \mathbf{C}$ such that

$$
\|U\|_{r, T_{\rho}} \leq K_{\mathbf{C}, S, \overline{\mathbf{C}}}^{(r)}(1+\rho)^{2 r}\|h\|_{r, T_{\rho}} .
$$

It is obvious that both $W$ and $U=W \circ \xi^{-1}$ depend linearly on $h$.

Case B. Assume now $\mathbf{C}<0, l<\infty, \overline{\mathbf{C}} / \mathbf{C} \geq l+1$ and $h$ has support in $|R| \leq \rho_{0}$. Let $\bar{H}, \bar{F}$ and $\bar{W}$ be the restrictions of $H, F$ and $W$ on $[-2 \pi, 2 \pi] \times \mathbf{R}$ respectively. By construction, $\bar{H}$ has compact support and it satisfies $\|\bar{F}\|_{l} \leq\|\bar{H}\|_{l} /|\overline{\mathbf{C}}|$.

Observe that $\overline{\mathbf{C}}-\alpha \mathbf{C}<0$ for any $\alpha \leq l$. So the series $\bar{W}_{0}^{(\alpha)}$ are uniformly convergent and

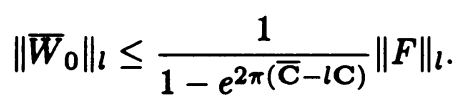

Therefore one can choose $K_{l}^{\prime}$ depending only on $l$, such that $\|\bar{W}\|_{l} \leq K_{l}\|\bar{H}\|_{l}$.

(4.12) Proposition. Let $\mathbf{C}, S, \mathbf{C}^{\prime}, \mathbf{C}^{\prime \prime}$ be nonzero real numbers such that $\mathbf{C}^{\prime} / \mathbf{C}$ and $\mathbf{C}^{\prime \prime} / \mathbf{C}$ are off $[0, l+1)$, where $l \in \mathbf{N} \cup\{\infty\}$, and let $g_{1}(R, \varphi, U, V)$, $g_{2}(R, \varphi, U, V)$ be $C^{\infty}$ functions defined on $\mathbf{R}^{4}$ with support in

$$
\Lambda_{\rho_{0}, \delta}=\left\{(R, \varphi, U, V) ;|R| \leq \rho_{0},|U| \leq \delta,|V| \leq \delta\right\} .
$$

Assume $g_{i}$ are periodic in the variable $\varphi$, of period $2 \pi$ and

$$
D^{\alpha} g_{i}(0, \varphi, U, V) \equiv 0 \quad \text { if }|\alpha| \leq l(i=1,2) .
$$

Then there exist $\mathbf{C}^{l}$ functions $U(R, \varphi), V(R, \varphi)$ defined on $\mathbf{R}^{2}$, which are solutions of

$$
\left\{\begin{array}{l}
\mathbf{C} R U_{R}+S U_{\varphi}=\mathbf{C}^{\prime} U+g_{1}(R, \varphi, U, V), \\
\mathbf{C} R V_{R}+S V_{\varphi}=\mathbf{C}^{\prime \prime} V+g_{2}(R, \varphi, U, V) .
\end{array}\right.
$$

Furthermore they are periodic of period $2 \pi$ in the variable $\varphi$ and satisfy $U(0, \varphi)=$ $V(0, \varphi)=0$.

PROOF. Let $\xi: \mathbf{R} \rightarrow \mathbf{R}$ be a $C^{\infty}$ bump function such that $\xi(x)=0$ if $|x|>1$ and $\xi(x)=1$ if $|x| \leq \frac{1}{2}$.

Let $\varepsilon>0$ be a small positive number. Define the following functions by recurrence on $i$ :

(i) $G_{j}=\xi\left(\varepsilon^{-1} R\right) g_{j}, j=1,2$,

(ii) $h_{0}^{1}=h_{0}^{2}=0$,

(iii) $U_{i+1}=L^{1}\left(h_{i}^{1}\right)$, where $L^{1}=L_{\mathbf{C}, S, \mathbf{C}}$, are as defined above,

(iv) $V_{i+1}=L^{2}\left(h_{i}^{2}\right)$, where $L^{2}=L_{\mathbf{C}, S, \mathbf{C}^{\prime \prime}}$,

(v) $h_{i}^{j}(R, \varphi)=G_{j}\left(R, \varphi, U_{i}(R, \varphi), V_{i}(R, \varphi)\right)$.

The proof of this proposition is quite lengthy however and involves many technicalities and estimations. We intend to provide the basic ingredients and the proof proceeds in eight steps.

Step 1. For $s<\infty, s \leq l+1$ one has $g_{i}=R^{s} \tilde{g}_{i}$ with $\tilde{g}_{i} \in\left(C_{2 \pi}^{l}\right)_{0}$.

Moreover, there are numbers $r \leq s$ and $\tilde{K}_{r, s}$ such that

$$
\left\|g_{i}\right\|_{r, \rho} \leq \tilde{K}_{r, s} \rho^{s-r} .
$$


On the other hand

$$
\left\|G_{i}\right\|_{r, \rho} \leq r !\|\xi\|_{r}\left(\sum_{j=0}^{r} \varepsilon^{-j}\left\|g_{i}\right\|_{r-j, \inf \{\varepsilon, \rho\}}\right) .
$$

Hence we obtain immediately that

$$
\left\|G_{i}\right\|_{r, \rho} \leq K_{r, s} \inf \{\varepsilon, \rho\}^{s-r} .
$$

Similar estimations can be done for

$$
\left\|\frac{\partial h_{i}}{\partial U}\right\|_{r, \rho}, \quad\left\|\frac{\partial h_{i}}{\partial V}\right\|_{r, \rho}, \quad\left\|G_{i}\right\|_{r}, \quad\left\|\frac{\partial G_{i}}{\partial U}\right\|_{r} \text { and }\left\|\frac{\partial G_{i}}{\partial V}\right\|_{r} \text {. }
$$

Step 2. (i) For any $r<l+1$, there exists $\varepsilon_{r}>0$ such that if $\varepsilon \leq \varepsilon_{r}$, then $\left\|U_{i}\right\|_{r, T_{\rho_{0}}} \leq \delta / 2$ and $\left\|V_{i}\right\|_{r, T_{\rho_{0}}} \leq \delta / 2$.

(ii) Assume $l=\infty$. For each $r$ there exists $\rho_{r}>0$, depending on $r, \varepsilon$ and $\xi$ such that for $\rho \leq \rho_{r}$ one has $\left\|U_{i}\right\|_{r, T_{\rho}} \leq \delta / 2$ and $\left\|V_{i}\right\|_{r, T_{\rho}} \leq \delta / 2$.

The assertions in Step 2 are proved in the following way.

$$
\left\|U_{1}\right\|_{r, T_{\rho_{0}}} \leq\left(1+\rho_{0}\right)^{2 r} K_{r}\left\|G_{1}^{0}\right\|_{r}, \quad\left\|V_{1}\right\|_{r, T_{\rho_{0}}} \leq\left(1+\rho_{0}\right)^{2 r} K_{r}\left\|G_{2}^{0}\right\|_{r},
$$

where $G_{j}^{0}(R, \varphi)=G_{j}(R, \varphi, 0,0)$ and $K_{r}$ is a positive number greater than $K_{\mathbf{C}, S, \mathbf{C}^{\prime}}^{(r)}$, $K_{\mathbf{C}, S, \mathbf{C}^{\prime \prime}}^{(r)}, K_{\mathbf{C}, S, \mathbf{C}^{\prime}}^{(r)}$ and $K_{\mathbf{C}, S, \mathbf{C}^{\prime \prime}}$. From (4.14), taking $s=r+1$, one has $\left\|U_{1}\right\|_{r, T_{\rho_{0}}} \leq$ $\delta / 2$ and $\left\|V_{1}\right\|_{r, T_{\rho_{0}}} \leq \delta / 2$.

If $l=\infty$, then we have necessarily that $\mathbf{C}^{\prime} / \mathbf{C}$ and $\mathbf{C}^{\prime \prime} / \mathbf{C}$ are negative. So, from (4.2) we get

$$
\left\|U_{1}\right\|_{r, T_{\rho}} \leq(1+\rho)^{2 r} K_{r}\left\|G_{1}^{0}\right\|_{r, T_{\rho}}, \quad\left\|V_{1}\right\|_{r, T_{\rho}} \leq(1+\rho)^{2 r} K_{r}\left\|G_{2}^{0}\right\|_{r, T_{\rho}} .
$$

By virtue of (4.14), taking $s=r+1$ and $\rho$ small enough we get

$$
\left\|U_{1}\right\|_{r, T_{\rho}} \leq \delta / 2 \text { and }\left\|V_{1}\right\|_{r, T_{\rho}} \leq \delta / 2
$$

Assume now

$$
\left\|V_{i}\right\|_{r, T_{\rho_{0}}} \leq \delta / 2 \text { and }\left\|V_{i}\right\|_{r, T_{\rho_{0}}} \leq \delta / 2
$$

Because of Remark (4.1), we have

$$
\left\|h_{i}^{j}\right\|_{r, T_{\rho 0}} \leq N_{r}\left\|G_{j}\right\|_{r, \Lambda_{\rho_{0}}}(1+\delta)^{r} \text {. }
$$

Applying (4.2) and (4.14), we get $\left\|U_{i+1}\right\|_{r, T_{\rho_{0}}} \leq \delta / 2$. We obtain a similar majoration for $V_{i+1}$.

If $l=\infty$, we can also get the following inequalities:

$$
\begin{gathered}
\left\|U_{i+1}\right\|_{r, T_{\rho}} \leq K_{r} N_{r}(1+\rho)^{2 r}(1+\delta)^{r}\left\|G_{1}\right\|_{r, \Lambda_{\rho}}, \\
\left\|V_{i+1}\right\|_{r, T_{\rho}} \leq K_{r} N_{r}(1+\rho)^{2 r}(1+\delta)^{r}\left\|G_{2}\right\|_{r, \Lambda_{\rho}} .
\end{gathered}
$$

Observe finally that

$$
\Lambda_{\rho} \subset T_{\rho} \times[-\delta, \delta] \times[-\delta, \delta] .
$$

The proofs of the assertions contained in Steps 3 and 4 are straightforward.

Step 3. (i) For $r<l+1$, there exists $\varepsilon_{r}^{\prime}$ such that

$$
\begin{aligned}
& \left\|h_{i+1}^{1}-h_{i}^{1}\right\|_{r, T_{\rho 0}}+\left\|h_{i+1}^{2}-h_{i}^{2}\right\|_{r, T_{\rho 0}} \\
& \quad \leq \varepsilon^{1 / 2}\left(\left\|U_{i+1}-U_{i}\right\|_{r, T_{\rho_{0}}}+\left\|V_{i+1}-V_{i}\right\|_{r, T_{\rho_{0}}}\right) \quad \text { with } \varepsilon \leq \varepsilon_{r}^{\prime} .
\end{aligned}
$$


(ii) Consider $l=\infty$. Associated with any $r$ there exists $\rho_{r}^{\prime}>0$, depending on $\varepsilon$ and $\xi$, such that

$$
\begin{aligned}
& \left\|h_{i+1}^{1}-h_{i}^{1}\right\|_{r, T_{\rho}}+\left\|h_{i+1}^{2}-h_{i}^{2}\right\|_{r, T_{\rho}} \\
& \quad \leq \rho^{1 / 2}\left(\left\|U_{i+1}-U_{i}\right\|_{r, T_{\rho}}+\left\|V_{i+1}-V_{i}\right\|_{r, T_{\rho}}\right) \quad \text { with } \rho \leq \rho_{r}^{\prime} .
\end{aligned}
$$

Step 4. Denote by $\bar{U}_{i}, \bar{V}_{i}$ the restrictions of $U_{i}, V_{i}$ to $T_{\rho_{0}}$ respectively. Let $r_{0}<l+1$. If $\varepsilon$ is small enough, then the sequence $\left(\bar{U}_{i}\right)$ and $\left(\bar{V}_{i}\right)$ converge in $C_{2 \pi}^{r_{0}}\left(T_{\rho_{0}}\right)$ to $\bar{U}$ and $\bar{V}$ respectively. Moreover if $l=\infty$, then for each $r$ there is $\rho_{r}>0$ such that $\bar{U}$ and $\bar{V}$ are of class $C^{r}$ on $\Omega_{\rho_{r}}=\left[-\rho_{r}, \rho_{r}\right] \times \mathbf{R}$.

Step 5. For any $r<l+1$, there is $\rho_{r}>0$ such that the restrictions $U_{i}, V_{i}$ on $\Omega_{\rho_{r}}=\left[-\rho_{r}, \rho_{r}\right] \times \mathbf{R}$ converge to $\bar{U}, \bar{V}$ in $C_{2 \pi}^{l}\left(\Omega_{\rho_{r}}\right)$ respectively. This follows directly from the above steps.

Step 6. It is easy to see that the images of $\bar{U}$ and $\bar{V}$ are contained in $[-\delta / 2, \delta / 2]$.

Step 7. Any solution $(U(R, \varphi), V(R, \varphi))$ of (4.13) defined and of class $C^{r}$ on an open set $\Omega$ can be extended to a unique solution defined and of class $C^{r}$ on $\bar{\Omega}$, where $\bar{\Omega}$ is the saturation of $\Omega$ by the flow of the vector field $Z=\mathrm{C} R \partial / \partial R+S \partial / \partial \varphi$ and by the action of the translation $(R, \varphi) \rightarrow(R, \varphi+2 \pi)$. Moreover, if $\Omega$ is a neighborhood of the $\varphi$-axis and $U$ and $V$ are periodic (in $\varphi$ ), then the respective extensions are also periodic.

We are going to demonstrate the assertion in Step 7. As above, consider the change of coordinates $\xi(R, \varphi)=\left(R e^{\mathbf{C} \varphi}, \varphi\right)$ which transforms $Z$ in $\partial / \partial \varphi$ and system (4.13) in

$$
\bar{U}_{\varphi}=\mathbf{C}^{\prime} \bar{U}+\bar{g}_{1}(R, \varphi, \bar{U}, \bar{V}), \quad \bar{V}_{\varphi}=\mathbf{C}^{\prime \prime} \bar{V}+\bar{g}_{2}(R, \varphi, \bar{U}, \bar{V}),
$$

where $\bar{U}=U \circ \xi, \bar{V}=V \circ \xi$ and $\bar{g}_{i}(R, \varphi, \bar{U}, \bar{V})=g_{i}\left(R e^{\mathbf{C} \varphi}, \varphi, U, V\right)$.

We may interpret this system as a vector field on $\mathbf{R}^{4}$ having the expression

$$
Z^{\prime}(R, \varphi, \bar{U}, \bar{V})=\left(\mathbf{C} \bar{U}+\bar{g}_{1}\right) \frac{\partial}{\partial \bar{U}}+\left(\mathbf{C}^{\prime \prime} \bar{V}+\bar{g}_{2}\right) \frac{\partial}{\partial \bar{V}}+\frac{\partial}{\partial \varphi} .
$$

The flow $\psi_{t}$ of $Z^{\prime}$ satisfies

$$
\left|\psi_{t}^{j}-\psi_{0}^{j}\right| \leq 2 e^{\overline{\mathbf{C}} \varphi}\left(1+\int_{0}^{\varphi} M e^{-\mathrm{C} s} d s\right), \quad j=3,4,
$$

where $\psi_{t}^{j}$ are the components of $\psi_{t}, M$ is a majorant $\left(\left|g_{1}\right|+\left|g_{2}\right|\right)$ and $\mathbf{C} \geq\left|\mathbf{C}^{\prime}\right|+\left|\mathbf{C}^{\prime \prime}\right|$.

The curves $\gamma_{R}: \varphi \rightarrow(R, \varphi, U \circ \xi(R, \varphi), V \circ \xi(R, \varphi))$ are, of course, integral curves of $Z^{\prime}$. From the unicity of solutions, we get

$$
\left\{\begin{array}{l}
U \circ \xi\left(R, \varphi_{1}+\varphi_{2}\right)=\psi_{\varphi_{2}}^{3}\left(R, \varphi_{1}, U \circ \xi\left(R, \varphi_{1}\right), V \circ \xi\left(R, \varphi_{1}\right)\right), \\
V \circ \xi\left(R, \varphi_{1}+\varphi_{2}\right)=\psi_{\varphi_{2}}^{4}\left(R, \varphi_{1}, U \circ \xi\left(R, \varphi_{1}\right), V \circ \xi\left(R, \varphi_{1}\right)\right) .
\end{array}\right.
$$

Let $\Omega^{\prime}=\xi^{-1}(\Omega)$ and let $\bar{\Omega}^{\prime}$ be the saturation of $\Omega^{\prime}$ by the vector field $\partial / \partial \varphi=$ $\xi^{*}(Z)$. As before we can extend $\bar{U}$ and $\bar{V}$ uniquely on $\bar{\Omega}^{\prime}$, preserving the class of differentiability.

Assume that $\Omega$ is a neighborhood of the $\varphi$-axis and $\bar{\Omega}=\mathbf{R}^{2}$. We want to demonstrate that the extensions $U^{\prime}=\bar{U}^{\prime} \circ \xi^{-1}, V^{\prime}=V^{\prime} \circ \xi$ are periodic. So we have to prove that they verify relation (4.3). 
Of course, $Z^{\prime}$ is invariant under the action of

$$
\Theta:(R, \varphi, U, V) \rightarrow\left(R e^{-2 \pi \mathrm{C}}, \varphi+2 \pi, U, V\right) .
$$

This implies that

$$
\psi_{t}^{j}\left(R e^{-2 \pi \mathrm{C}}, \varphi+2 \pi, U, V\right)=\psi_{t}^{j}(R, \varphi, U, V), \quad j=3,4 .
$$

Now, by combining (4.17) and (4.18) we conclude the present proof.

The next result is an immediate consequence of the last step:

Step 8. Any $C^{r}$ solution of (4.13) in $\Omega_{\rho_{r}}$ can be extended to a unique $C^{r}$ solution defined on $\mathbf{R}^{2}$.

Now the conclusion of Proposition (4.12) is obvious.

PROOF OF THEOREM 2. We are going to determine a diffeomorphism $f$ on a neighborhood of the $\varphi$-axis of the type

$$
f(R, \varphi)=\left\{\begin{array}{l}
r(R, \varphi)=R+R^{p+1} u(R, \varphi) \\
\Theta(R, \varphi)=\varphi+R^{p} v(R, \varphi)
\end{array}\right.
$$

which is a conjugacy between

$$
\dot{r}=\mathbf{C} r^{k+1}+r^{k+2} a(r, \theta), \quad \dot{\boldsymbol{\Theta}}=S r^{k}+r^{k+1} b(r, \theta)
$$

and

$$
\dot{R}=\mathbf{C} R^{k+1}, \quad \dot{\varphi}=S R^{k} .
$$

Because of Theorem 1, one may of course assume the following forms of $a$ and $b$

$$
a(r, \theta)=r^{k-1} \alpha(r, \theta) \text { and } b(r, \theta)=r^{k-1} \beta(r, \theta) .
$$

Putting (4.19) and (4.21) in (4.20) we obtain

$$
\left\{\begin{array}{l}
\mathbf{C} R u_{R}+S u_{\varphi}=\mathbf{C}^{\prime} u+R^{p} u^{2} \tau_{1}\left(R^{p} u\right)+a_{1}\left(R+R^{p+1} u, \varphi+R^{p} v\right) \\
\mathbf{C} R v_{R}+S v_{\varphi}=\mathbf{C}^{\prime \prime} u+u \tau_{2}\left(R^{p} u\right)+b_{1}\left(R+R^{p+1} u, \varphi+R^{p} \varphi\right)
\end{array}\right.
$$

where

$$
\begin{aligned}
& \mathbf{C}^{\prime}=(k-p) \mathbf{C}, \quad \mathbf{C}^{\prime \prime}=-p \mathbf{C}, \\
& \tau_{1}(Z)=\mathbf{C} Z^{-2}\left[(1+Z)^{k+1}-1-(k+1) Z\right], \\
& \tau_{2}(Z)=S Z^{-1}\left[(1+Z)^{k}-1\right], \\
& a_{1}=R^{1-p}\left(1+R^{p+1} u\right)^{k+2} a \text { and } b_{1}=R^{2-p}\left(1+R^{p+1} u\right)^{k+1} b .
\end{aligned}
$$

By a change of variables $U, V$, we may consider (4.22) in the form

$$
\left\{\begin{array}{l}
\mathbf{C} R U_{R}+S U_{\varphi}=\mathbf{C}^{\prime} U+a_{2}(R, \varphi, U, V), \\
\mathbf{C} R V_{R}+S V_{\varphi}=\mathbf{C}^{\prime \prime} V+b_{2}(R, \varphi, U, V),
\end{array}\right.
$$

where $a_{2}, b_{2}$ are $C^{\infty}$-functions defined on a neighborhood of the $\varphi$-axis in $\mathbf{R}^{4}$, periodics of period $2 \pi$ in the variable $\varphi$, and $a_{2}=R^{k-p} \tilde{a}_{2}, b_{2}=R^{k-p} \tilde{b}_{2}$ with $\tilde{a}_{2}$, $b_{2}$ being of class $C^{\infty}$.

Observe that, if both $A=r^{k+2} a, B=r^{k+1} b$ are $\infty$-flat on the $\varphi$-axis, then $a_{2}, b_{2}$ will be $\infty$-flat on the hyperplane $R=0$. 
Now take $u=u(R, U), v=v(R, U, V)$ to be functions defined implicitly by the equations

$$
U=u e^{H_{1}(R, u)}, \quad V=v+H_{2}(R, u)
$$

with $H_{1}, H_{2}$ unknown.

Now we put (4.24) in (4.23) and try to determine $H_{1}, H_{2}, a_{2}$, and $b_{2}$ to obtain (4.22). So

$$
\begin{aligned}
a_{2}(R, \varphi, U, V)= & e^{H_{1}(r, u)} a_{1}\left(R+R^{p+1} u, \varphi+R^{p} v\right), \\
b_{2}(R, \varphi, U, V)= & b_{1}\left(R+R^{p+1} u, \varphi+R^{p} v\right)+\left(H_{2}\right)_{u}(R, u) \\
& \cdot a_{1}\left(R+R^{p+1} u, \varphi+R^{p} v\right) .
\end{aligned}
$$

Observe that $H_{1}, H_{2}$ must satisfy the following equations:

$$
\begin{gathered}
\left(\mathbf{C}^{\prime} U+R^{p} u^{2} \tau_{1}\left(R^{p} u\right)\right)\left(u\left(H_{1}\right)_{u}+1\right)-\mathbf{C}^{\prime} u+\mathbf{C} R u\left(H_{1}\right)_{R}=0, \\
\left(\mathbf{C}^{\prime \prime} H_{2}-\mathbf{C} R\left(H_{2}\right)_{R}-\left(\mathbf{C}^{\prime} u+R^{p} u^{2} \tau_{1}\left(R^{p} u\right)\left(H_{2}\right)_{u}\right)\right)=u \tau_{2}\left(R^{p} u\right) .
\end{gathered}
$$

Put

$$
H_{1}(R, u)=L_{1}\left(R^{p} u\right), \quad H_{2}(R, u)=u L_{2}\left(R^{p} u\right)
$$

with $L_{1}(Z), L_{2}(Z)$ being $C^{\infty}$ functions of one variable.

The above equations take the following forms:

$$
\begin{gathered}
\frac{d L_{1}}{d Z}=\frac{-Z \tau_{1}(Z)}{k \mathbf{C}+Z}, \\
L_{2}(Z)+Z \frac{d L_{2}}{d Z}(Z)=\frac{-\tau_{2}(Z)}{k \mathbf{C}+Z \tau_{1}(Z)} .
\end{gathered}
$$

Observe that the right side of equation $\left(4.26^{\prime}\right)$ is analytic. So, we can integrate the above equations without difficulties and determine the desired $H_{1}, H_{2}, a_{2}$, and $b_{2}$.

Suppose now $a_{2}$ and $b_{2}$ are defined on

$$
\Omega_{R_{0}, \delta_{0}}=\left[-R_{0}, R_{0}\right] \times \mathbf{R} \times\left[-\delta_{0}, \delta_{0}\right]^{2}
$$

and are periodic of period $2 \pi$ in the variable $\varphi$.

Let $\lambda: \mathbf{R} \rightarrow \mathbf{R}$ be a $C^{\infty}$ bump function with support on $\left[-\varepsilon_{0}, \varepsilon_{0}\right]$ and such that $\lambda(x)=1$ if $x \in\left[-\varepsilon_{0} / 2, \varepsilon_{0} / 2\right], \varepsilon_{0} \leq \inf \left(R_{0}, \delta_{0}\right)$.

Define

$$
a_{3}=\lambda(R) \lambda(U) \lambda(V) a_{2} \quad \text { and } b_{3}=\lambda(R) \lambda(U) \lambda(V) b_{2} .
$$

In (4.23) we replace $a_{2}, b_{2}$ and $a_{3}, b_{3}$ respectively and apply Proposition (4.12). If the normal form of (4.20) is (4.21), we may of course assume that $a$ and $b$ are $\infty$-flat on the $\theta$-axis. So we may consider $a_{2}, b_{2}$ as being $\infty$-flat on $R=0$. Hence if we set $p<k$ then system (4.22) satisfies the assumptions of Proposition (4.12).

In the general case, we verify directly that if $l \leq k-p-1$, then $\mathbf{C}^{\prime} / \mathbf{C} \geq l+1$, $\mathbf{C}^{\prime \prime} / \mathbf{C}<0$ and $a_{2}, b_{2}$ are $l$-flat along $R=0$.

Now the conclusion of the theorem is direct. 


\section{REFERENCES}

1. F. Dumortier, Singularities of vector fields on the plane, J. Differential Equations 23 (1977), 53-106.

2. F. Dumortier and R. Roussarie, Germes de diffémorphismes et de champs de vecteurs en classe de différentiabilité finie, Ann. Inst. Fourier (Grenoble) 33 (1983), 195-267.

3. J. Hale, Ordinary differential equations, Wiley, New York, 1969.

4. F. Takens, Forced oscillations and bifurcations, Comm. Math. Institute, vol. 3, Utrecht University, 1974.

5. _ Normal forms for certain singularities of vector fields, Ann. Inst. Fourier (Grenoble) 23 (1973), 163-165.

6. M. A. Teixeira, A topological invariant for discontinuous vector fields, Nonlinear Analysis: Theory, Methods and Applications (to appear).

7. S. P. Tokarev, Smooth equivalence of differential-equation systems in the plane in the case of a noncoarse focus, Differentisial'nye Uravnenija 13 (1977), 613-617.

Universite de toulouse 1, Place anatole France, 31042 Toulouse Cedex, France

IMECC-UNICAMP, CaIXA Postal 1170, CAMPINAS-SP, BRAsIL 